Research Paper

\title{
The Expression of CD9 and PIK3CD is Associated with Prognosis of Follicular Lymphoma
}

\author{
Tieying Dong ${ }^{* 1}$, Zhaoliang Liu ${ }^{* 1}$,2, Shu Zhao ${ }^{1}$, Chengyi Hu, Yupeng Liu, WenJie Ma1 ${ }^{1}$ Qingyuan Zhang1\#® \\ 1. Department of Internal Medicine, The Third Affiliated Hospital of Harbin Medical University, Haping Road 150 of Nangang District, Harbin, Heilongjiang \\ Province 150081, China \\ 2. Cancer Research Institute, Harbin Medical University, Harbin, China \\ 3. Cancer Research Institute of Heilongjiang, Harbin, China \\ \# This experiment was performed in the Oncology Key Lab of the Department of Higher Education of Heilongjiang Province Institution of Higher Education. \\ * These authors contributed equally to this work and should be considered co-first authors. \\ $\triangle$ Corresponding author: E-mail: dongty@hrbmu.edu.cn
}

() 2015 Ivyspring International Publisher. Reproduction is permitted for personal, noncommercial use, provided that the article is in whole, unmodified, and properly cited. See http:/ / ivyspring.com/terms for terms and conditions.

Received: 2014.12.08; Accepted: 2015.06.12; Published: 2015.10.15

\begin{abstract}
Background: Follicular lymphoma (FL) frequently develops drug-resistance and transforms into more aggressive subtypes over time. It is urgent to find prognostic biomarkers and disclose signaling pathways that have potential to be drug targets. In this study, we investigated the association of FL prognosis with the expression of 2 proteins: PIK3CD, a PI3K pathway component, and CD9, a tetraspanin family member.

Method: The expression of PIK3CD and CD9 were examined on $76 \mathrm{FL}$ tumor tissues and 15 normal tissues with Immunohistochemistry. Chi-square test, Cox proportional hazards model, and Kaplan-Meier estimates were used to analyze the relationships of CD9 and PIK3CD expression and major clinicopathological features.

Result: PIK3CD expression was significantly higher, whereas CD9 expression was significantly lower in the $76 \mathrm{FL}$ specimens than normal tissues. Concomitantly, low CD9 or high PIK3CD expression is associated with high degrees of Ann Arbor stages. In agreement with this, PIK3CD is an unfavorable and CD9 is a favorable factor for progression-free survival (PFS). Interestingly, PIK3CD expression is negatively correlated with CD9 expression, and the PIK3CD-high/CD9-low was significantly associated with short PFS when the 2 factors were combined together. Lastly, CD9 expression was significant higher in patients with bone marrow infiltration (BMI) than those without BMI.

Conclusion: Both CD9 and PIK3CD are prognostic markers of FL. The negative correlation between $C D 9$ and PIK3CD expression suggests that there may be crosstalks of the 2 proteins in FL.

Key words: CD9; PI3KCD; Follicular Lymphoma; Prognosis; Bone Marrow Infiltration

Follicular lymphoma (FL) is the most common indolent B-cell non-Hodgkin's lymphoma, generated from germinal center B cells [1]. It is characterized by the relatively low malignancy degree, slow progression and high response rates to therapy, such as rituximab [2-4]. However, FL is a heterogeneous disease and presents variable outcomes. About 30-40\%

FL will undergo a histological transformation and develop into more aggressive lymphomas, such as diffuse large B cell lymphoma (DLBCL). Consequently, this population of FL will have a poor outcome due to increasing drug resistance and multiple relapses [2, 5-8]. After comparing $12 \mathrm{FL}$ samples and their counter parts of DLBCL by microarray, Lossos IS
\end{abstract}

\section{Introduction}


et al found that no single genes are subject to the same changes during disease progression [9]. These results suggested that FL progression is complicate and may need multiple alternative mechanisms. Therefore, stratification of FL subpopulations is required to evaluate their clinic outcomes, and more biomarkers are in need to identify the subpopulations with poor prognosis.

In B cells, B-cell antigen receptor (BCR) is a ubiquitous surface protein. BCR signaling regulates multiple B cell biological functions, such as growth, proliferation, differentiation and survival [10-13], thus it is a key element of B-cell survival and lymphomagenesis [14]. Intracellular signal transduction of BCRs is almost invariably dependent on phosphoinositide 3-kinase (PI3K) signaling [15]. PI3Ks catalyze the production of lipid second messengers in cells, primarily phosphatidylinosital 3,4,5-trisphosphate (PIP3) $[16,17]$. The class I PI3Ks are composed of a regulatory subunit and a p110 catalytic subunit. There are 4 isoforms of the catalytic subunits of Class I PI3Ks: PIK3CA, PIK3CB, PIK3CG, and PIK3CD, of which PIK3CD (also named with PI3K $\delta, p 110 \delta$ ) is primarily expressed in leukocytes and may account for $60 \%$ of PI3K activity in B cells [18-20]. Ablation of PIK3CD in mice blocks pro-B cell differentiation and decreased numbers of mature B cells in the circulation, suggesting an indispensible role in B-cell follicular maturation and survival. Inhibitors that block pan-PI3K or PIK3CD activity have shown promising results in animal experiments or clinic trails for treatment of variable lymphoma including FL and lymphoid and myeloid leukemia [17, 21, 22]. Though class I PI3K aberrancy has been frequently implicated in many forms of B-cell malignancy, such as chronic lymphocytic leukemia (CLL), mantel cell lymphoma (MCL), and DLBCL, it is not well studied in FL [23]. In FL, a subset of patients shows activation of PI3K, demonstrated by phosphorylation of Akt, a downstream kinase of PI3Ks [21, 24]. However, it is not clear whether PI3K pathway activity is associated with FL prognoses.

Proper BCR signaling also requires Bruton tyrosine kinase (BTK) whose activation is dependent on the production of PIP3 by PI3Ks. One of Btk targets in $B$ cells is CD9, identified by Btk mutant and knockout mice [25]. CD9 (also known as motility-related protein-1, MRP-1) is a $24-\mathrm{kDa}$ cell membrane glycoprotein that belongs to the tetraspanin family characterized by 4 transmembrane domains [26]. Partnering with integrins, immunoglobulin superfamily receptors, and other tetraspanin proteins, CD9 forms specialized membrane microdomains on the cell surface and transduces extracellular signals inside to promote apopotosis, suppress metastasis, and modulate cell adhesion [27-31]. Indolent B lymphoma cells depend on the interaction of CD9 and follicular dendritic cells (FDC) to survive [27]. However, when the disease progresses, tumor cells lose CD9 expression and bypass the pathway [24, 25, 32, 33]. Although reduced CD9 expression was associated with less favorable prognosis in multiple cancers [34-36], it still remains elusive whether CD9 plays a role in FL progression.

In this study, we examined the expression of PIK3CD and CD9 in FL to investigate their relationships with FL prognoses. We found both CD9 and PIK3CD expression are associated with FL prognoses, and their expression is negatively correlated in FL.

\section{Materials and Methods}

\section{Patients and tissue samples}

Formalin-fixed paraffin-embedded FL sections were obtained from The Third Affiliated Hospital of Harbin Medical University. The patients were firstly diagnosed as FL without any severe systemic disease or combined tumor between October 2006 and May 2013, and didn't receive any treatment before diagnosis. Patients were excluded from the study if they could not bear first-line chemotherapy due to pregnancy, breast feeding, or poor compliance, or if they were less than 18 years of age. Total 76 surgical FL samples were collected. The cohort includes 32 males and 44 females, and the average age of these patients is 50.90 (ranged from 25 to 76). According to Ann Arbor Stages, 29 were in stage I or II, 47 in stage III or IV. Stage IV contains 24 patients, and all of them had bone marrow infiltration but not other extra-nodal organs infiltration. The progression-free survival (PFS) ranged from 4 months to 35 months, and average PFS was 16.3 months. Randomly-selected 15 normal samples during the same period were used as controls. This study was conducted with approval from the Ethics Committee of the Third Affiliated Hospital of Harbin Medical University.

\section{Immunohistochemistry (IHC)}

Four micrometers of paraffin sections were used for immunohistochemical staining. They were dewaxed, incubated in saline sodium citrate $(\mathrm{pH}=$ 7.0) for $2 \mathrm{~min}$ in pressure heating environment for antigen retrieval, then soaked in $3 \% \mathrm{H} 2 \mathrm{O} 2$ solution for 25 min. Rabbit monoclonal CD9 antibody or mouse monoclonal PIK3CD antibody (both purchased from OriGene Technologies, Maryland, USA) was applied overnight with a dilution of $1: 200$ at $4^{\circ} \mathrm{C}$, and the sections were incubated with secondary antibody at room temperature for $25 \mathrm{~min}$, then visualized by DAB.

The stained specimens were reviewed by two pathologists independently. At least five visual fields 
were observed for each section under high power lens (400X) to calculate the percentage of positive cells. The sections were then scored semi-quantitatively for CD9 as the following criterions: classified as $0(<10 \%)$, $1(10 \%$ to $20 \%), 2(20 \%$ to $30 \%), 3(30 \%$ to $40 \%)$ and 4 ( $>40 \%$ ); intensities were classified as 0 (no staining), 1(weak staining), 2 (distinct staining), and 3 (strong staining). Specimen with a total score (percentage score multiplied intensity score) $>3$ were classified as CD9-positive $(+)$, otherwise were CD9-negtive (-). PIK3CD was scored as same intensity criterion as CD9, but the 0 and 1 scores were classified as PIK3CD-negtive (-), 2 and 3 scores as PIK3CD-positive $(+)$.

\section{Statistical analysis}

All the data were analyzed with SPSS20.0 software and chi square tests were used to compare the differences of the CD9 and PIK3CD expression between the FL and the normal tissues. The correlations of the CD9 and PIK3CD expression levels were also analyzed using chi square tests. Survival curves were generated with the Kaplan-Meier method, and Cox's proportional hazards regression model was used to evaluate the hazards. Differences with $\mathrm{P}<0.05$ were considered statistically significant.
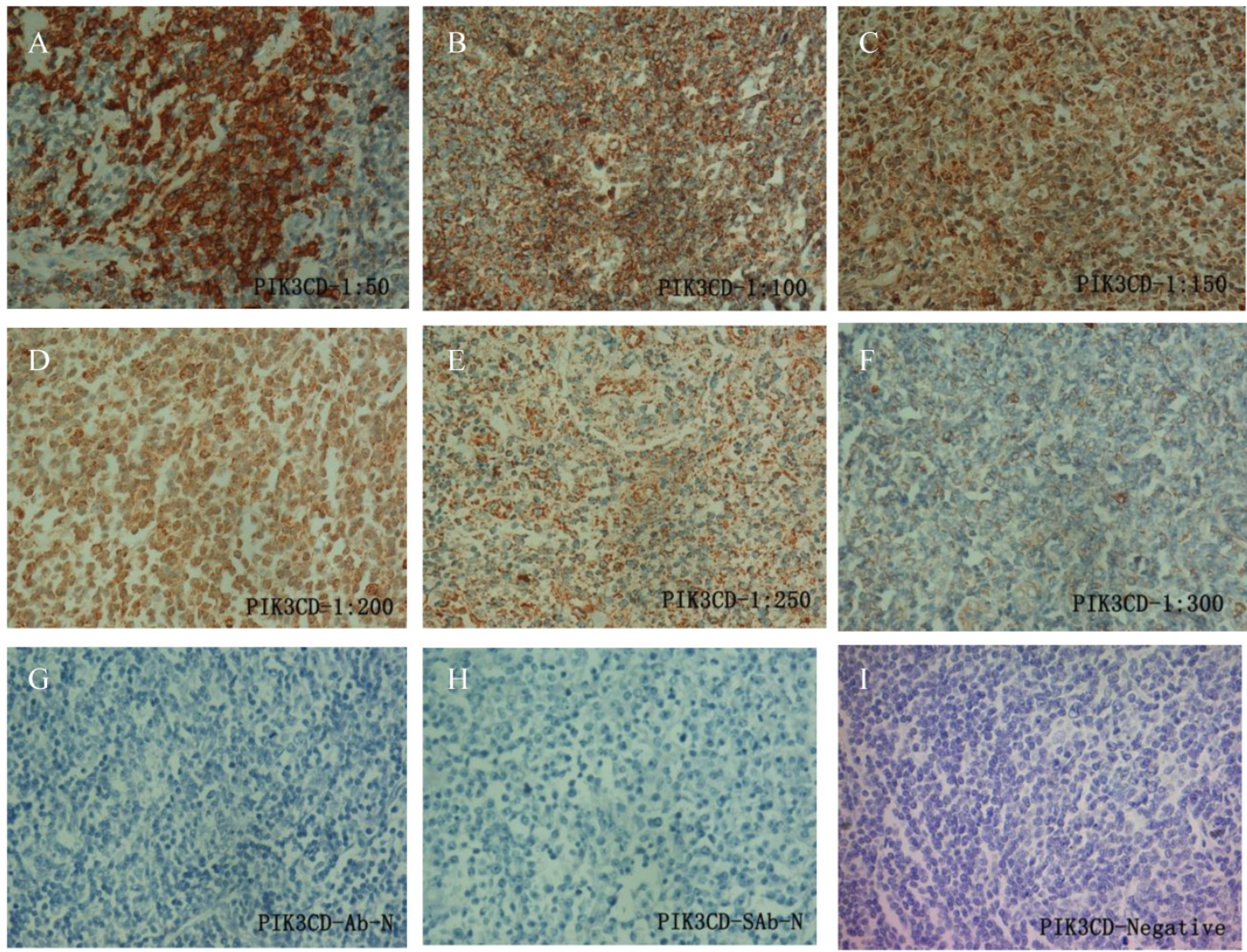

Fig. 1. IHC of PIK3CD in FL patients. A series of PIK3CD antibody dilutions were used to determine the specificity of the antibody: A, 1:50; $B$, 1:100; C: 1:150; D, 1:200; E, 1:250; F, 1:300. Sections without primary PIK3CD antibody (G) or without secondary antibody (H) were used as negative control. A representative PIK3CD negative patient (I) was also shown. 
Table 1. The expression of CD9 and PIK3CD in $76 \mathrm{FL}$ patients and their association with clinicopathological features.

\begin{tabular}{|c|c|c|c|c|c|c|c|}
\hline \multicolumn{2}{|c|}{ Pathological characteristics in FL } & \multicolumn{2}{|c|}{ CD9 Expression } & \multirow[t]{2}{*}{$\mathrm{P}$} & \multicolumn{2}{|c|}{ PIK3CD Expression } & \multirow[t]{2}{*}{$\mathrm{P}$} \\
\hline & & Positive & Negative & & Positive & Negative & \\
\hline \multirow[t]{2}{*}{ Gender } & Male & $8(25.0 \%)$ & $24(75.0 \%)$ & 0.394 & $24(75.0 \%)$ & $8(25.0 \%)$ & 0.824 \\
\hline & Femal & $15(34.1 \%)$ & $29(65.9 \%)$ & & $32(72.7 \%)$ & $12(27.3 \%)$ & \\
\hline \multirow[t]{2}{*}{ Age } & $\geq 60$ years & $7(28.0 \%)$ & $18(72.0 \%)$ & 0.764 & $19(76.0 \%)$ & $6(24.0 \%)$ & 0.748 \\
\hline & $<60$ years & $16(31.4 \%)$ & $35(68.6 \%)$ & & $37(72.5 \%)$ & $14(27.5 \%)$ & \\
\hline \multirow[t]{2}{*}{ Ann Arbor Stages } & $\mathrm{I}-\mathrm{II}$ & $10(34.5 \%)$ & $19(65.5 \%)$ & 0.009 & $20(69.0 \%)$ & $9(31.0 \%)$ & 0.022 \\
\hline & III - IV & $13(27.7 \%)$ & $34(72.3 \%)$ & & $38(77.6 \%)$ & $11(22.4 \%)$ & \\
\hline \multirow[t]{2}{*}{ Pathological Stages } & $\mathrm{I}-\mathrm{II}$ & $17(27.4 \%)$ & $45(72.6 \%)$ & 0.256 & $49(79.0 \%)$ & $13(21.0 \%)$ & 0.241 \\
\hline & III & $6(42.9 \%)$ & $8(57.1 \%)$ & & $9(64.3 \%)$ & $5(35.7 \%)$ & \\
\hline \multirow[t]{4}{*}{ FLIPI Risk } & Low Risk & $15(31.2 \%)$ & $33(68.8 \%)$ & 0.745 & $33(68.8 \%)$ & $15(31.2 \%)$ & 0.534 \\
\hline & $\begin{array}{l}\text { Low To Intermediate } \\
\text { Risk }\end{array}$ & $3(20.0 \%)$ & $12(80.0 \%)$ & & $13(86.7 \%)$ & $2(13.3 \%)$ & \\
\hline & $\begin{array}{l}\text { Intermediate To High } \\
\text { Risk }\end{array}$ & $4(40.0 \%)$ & $6(60.0 \%)$ & & $8(80.0 \%)$ & $2(20.0 \%)$ & \\
\hline & High Risk & $1(33.3 \%)$ & $2(66.7 \%)$ & & $2(66.7 \%)$ & $1(33.3 \%)$ & \\
\hline \multirow[t]{2}{*}{ Group } & FL & $23(30.26 \%)$ & $53(69.74 \%)$ & $<0.001$ & $56(73.68 \%)$ & $20(26.32 \%)$ & $<0.001$ \\
\hline & Normal & $10(66.67 \%)$ & $5(33.33 \%)$ & & $4(26.67 \%)$ & $11(73.33 \%)$ & \\
\hline
\end{tabular}

When comparing different clinicopathological characteristics, PIK3CD expression didn't differ between groups with pathological Stages or Follicular Lymphoma International Prognostic Indexes (FLIPI)[39] (Table 1). However, PIK3CD expression is significantly higher in patients of Ann Arbor stages III to IV $(77.6 \%, 38 / 47)$ than stages I to II $(69.0 \%, 20 / 29)$ (Table 1). More importantly, PIK3CD expression is negatively related with the length of progression-free survivals (PFS) (Fig. 4A). The median PFS of the PIK3CD $(+)$ patients was 12.5 months with a range from 4 month to 34 months, whereas the median PFS of the PIK3CD(-) patients was 24 months with a range from 12 month to 35 months $(\mathrm{P}<0.001, \mathrm{HR}=2.829$, 95.0\% CI: 1.634-4.897) (Fig. 4A).

\section{CD9 expression is significantly low in FL and associated with Ann Arbor stages and PFS}

Similarly as PIK3CD antibody, we first tested CD9 antibody with a series of antibody dilutions and proper negative controls, then we determined CD9 expression in our patient samples (Fig. 2). As previously reported, CD9 mainly exists on the cell membrane, but cytoplasmic signals were also observed [27] (Fig. 2). Similarly as PIK3CD, CD9 expression didn't differ according to the genders or ages, with $25.0 \%$ male and $34.1 \%$ of female patients, as well as $28.0 \%$ of more than and $31.4 \%$ less than 60 years old patients showing CD9 high expression (Table 1). However, CD9 expression was significantly reduced in FL patients comparing with normal controls. In the normal tissues, $66.67 \%(10 / 15)$ of specimens were categorized to $\mathrm{CD} 9(+)$ according to the methods described above while the positive rate of CD9 in FL was only $30.26 \%$ (23/76) (Fig. 3B, Table 1).

Although CD9 expression didn't show statistical differences in groups of pathological stages and Follicular Lymphoma International Prognostic Index
(FLIPI) (all $\mathrm{P}>0.05$ ), its expression is significantly lower in the Ann Arbor Stages III to IV than stages I to II groups (Table 1). Thirty-four point five percent of $(10 / 29)$ patients were CD9 $(+)$ in the Ann Arbor Stage I to II group whereas only $27.7 \%(13 / 47)$ patients were CD9(+) in Ann Arbor Stages III and IV (P=0.009) (Table 1). More importantly, reduced CD9 expression predicted shorter PFS (Fig. 4B). PFS of CD9(+) patients ranged from 4 months to 35 months, and the median PFS was 19 months. Meanwhile, PFS of CD9(-) ranged from 4 month to 26 months and the median PFS was 15 months $(\mathrm{P}=0.001, \mathrm{HR}=0.385,95.0 \% \mathrm{CI}: 0.241-0.693)$ (Fig. 4B). These results suggested that CD9 expression negatively correlated with FL prognostic outcomes and may be attributed FL progression.

\section{CD9 expression is negatively correlated with PIK3CD expression, and predicts PFS coop- eratively}

There are evidences that suggested CD9 and PI3K pathway crosstalks [25, 40]. Therefore, we analyzed the correlation between CD9 and PIK3CD expression. Inside the cohort, CD9 and PIK3CD expression were categorized 4 groups: the $\mathrm{CD} 9(+) / \mathrm{PIK} 3 \mathrm{CD}(+)$ group, which contained $15.8 \%$ $(12 / 76)$ patients; the CD9(+)/PIK3CD $(-)$ group which contained $14.5 \%(11 / 76)$ patients; the CD9(-)/PIK3CD $(+)$ group which contained $57.9 \%$ (44/76) patients; and the CD9(-)/PIK3CD(-) group which contained $11.8 \%$ (9/76) patients (Table 2). From table 2, we can see when PIK3CD expression is high (totally 56 cases), CD9 expression is always low $(44 / 56)$. On the contrary, when CD9 was low (totally 53 cases), PIK3CD expression is always high (44/53). Statistical analysis revealed that CD9 expression was negatively correlated with PIK3CD expression in FL $(p=0.005$, $\mathrm{R}=-0.322$ ). 
Table 2. PIK3CD expression was negatively correlated with CD9 expression in $\mathrm{FL}$

\begin{tabular}{lllll}
\hline & PIK3CD+ & PIK3CD- & P & $\mathrm{R}$ \\
\hline CD9+ & 12 & 11 & 0.005 & -0.322 \\
CD9- & 44 & 9 & & \\
\hline
\end{tabular}

Next, we analyzed the PFS of above 4 groups of patients. Our results showed that the median PFS of CD9 $(+) /$ PIK3CD (-) group is 27 months (ranged from 14 to 35 months), while that of CD9 (-)/PIK3CD (+) group is only 12.5 months (ranged from 4 to 26 months). The CD9(+)/PIK3CD(-) group has a significantly longer PFS than CD9(-)/PIK3CD $(+)$ group $(p<0.001)$ (Fig. 5). In multivariate analysis using cox risk regression model, we had not gotten useful data for CD9 or PIK3CD as an independent predictor for FL prognosis.

\section{CD9 expression promotes bone marrow infil- tration}

Bone marrow infiltration is a sign of metastasis, and associated with bad prognosis [41, 42]. Next we analyzed the relationship of CD9 and PIK3CD expression with FL bone marrow infiltration. In the cohort, all 24 Ann Arbor Stages IV patients were in hematopathy phase showing bone marrow infiltration at the first diagnosis, but were absent of other extra-nodal organs infiltration. Surprisingly, we found that CD9 expression was significantly higher in patients with bone marrow infiltration $(45.8 \%, 11 / 24)$ than those without bone marrow infiltration $(23.1 \%$, $12 / 52)(\mathrm{P}=0.045)$ (Table 3). Although CD9 expression is significantly correlated with PIK3CD expression, PIK3CD expression didn't associate with the status of bone marrow infiltration ( $\mathrm{P}=0.461$, Table 3$)$.

Table 3. CD9 Expression in FL was associated with bone marrow infiltration

\begin{tabular}{|c|c|c|c|c|c|c|c|}
\hline \multirow{2}{*}{\multicolumn{2}{|c|}{$\begin{array}{l}\text { Pathological charac- } \\
\text { teristics in FL }\end{array}$}} & \multicolumn{2}{|c|}{ CD9 Expression } & \multirow[t]{2}{*}{$\mathrm{P}$} & \multicolumn{3}{|c|}{ PIK3CD Expression $\mathrm{P}$} \\
\hline & & Positive & Negative & & Positive & Negative & \\
\hline Bone & Negative & $12(23.1 \%)$ & $40(76.4 \%)$ & 0.045 & $37(71.2 \%)$ & $15(28.8 \%)$ & 0.461 \\
\hline $\begin{array}{l}\text { Marrow } \\
\text { Infiltration }\end{array}$ & Positive & $11(45.8 \%)$ & $13(54.2 \%)$ & & $19(79.2 \%)$ & $5(20.8 \%)$ & \\
\hline
\end{tabular}
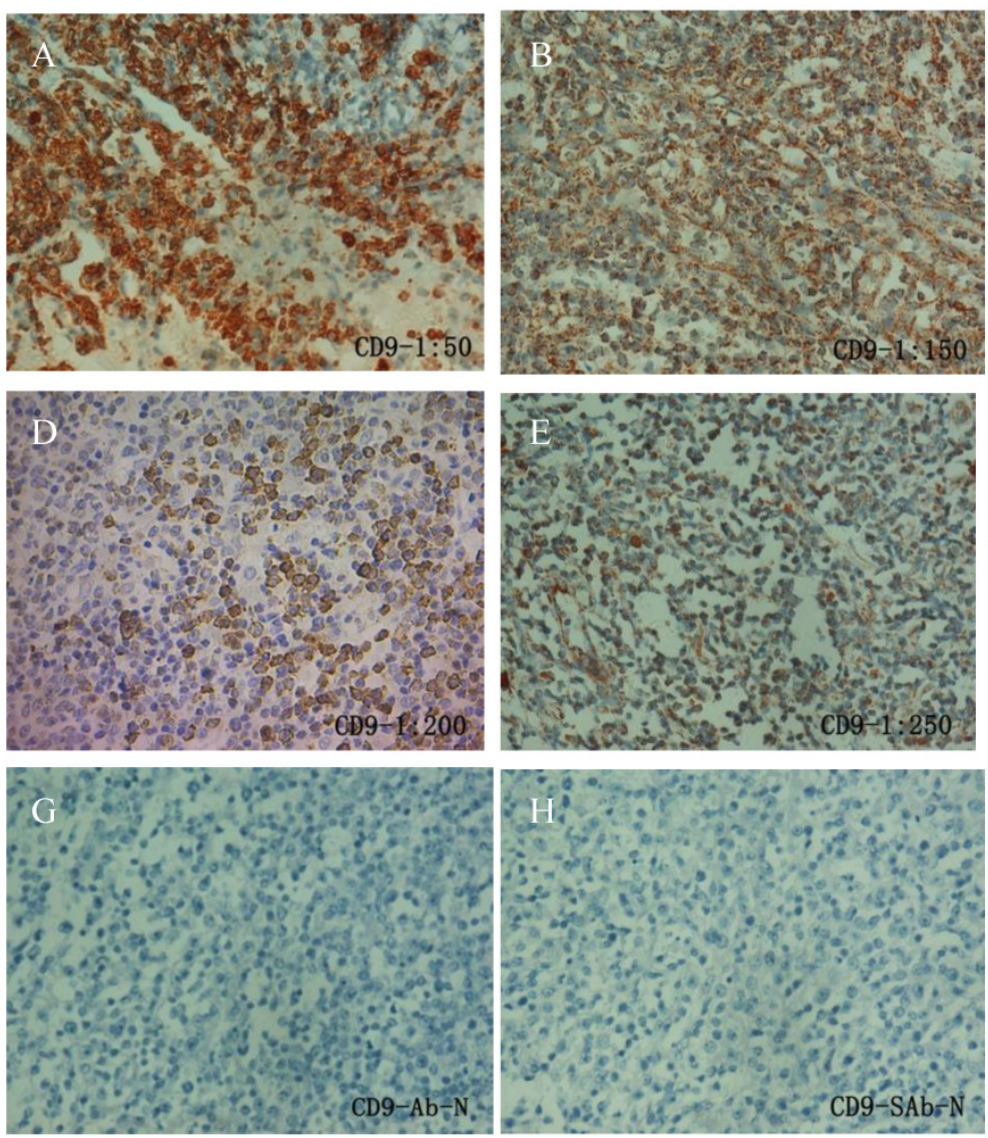
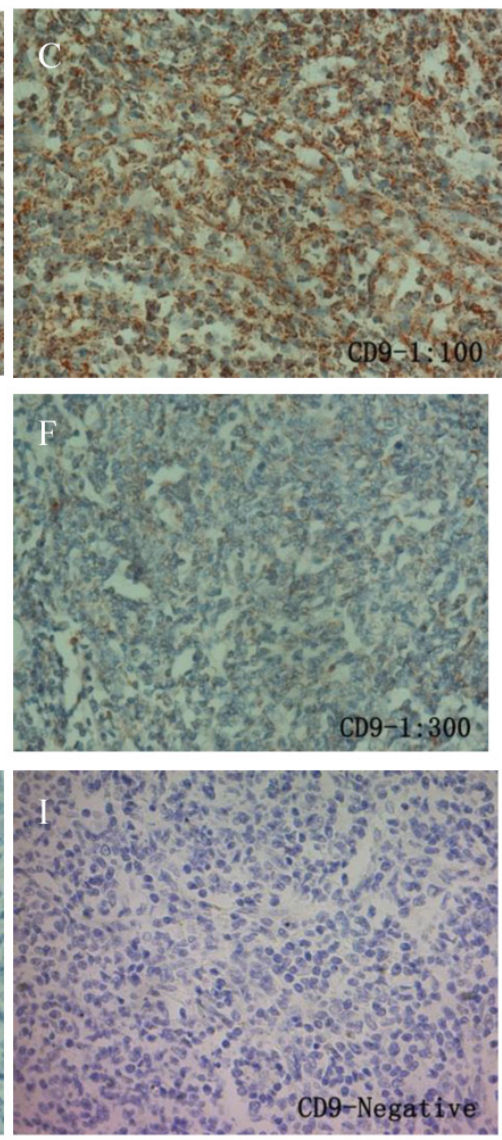

Fig. 2. IHC of CD9 in FL patients. A series of CD9 antibody dilutions were used to determine the specificity of the antibody: $A$, 1:50; $B$, 1:100; C: 1:150; D, 1:200; E, 1:250; F, 1:300. Sections without primary CD9 antibody $(G)$ or without secondary antibody $(H)$ were used as negative control. $A$ representative CD9 negative patient (I) was also shown. 

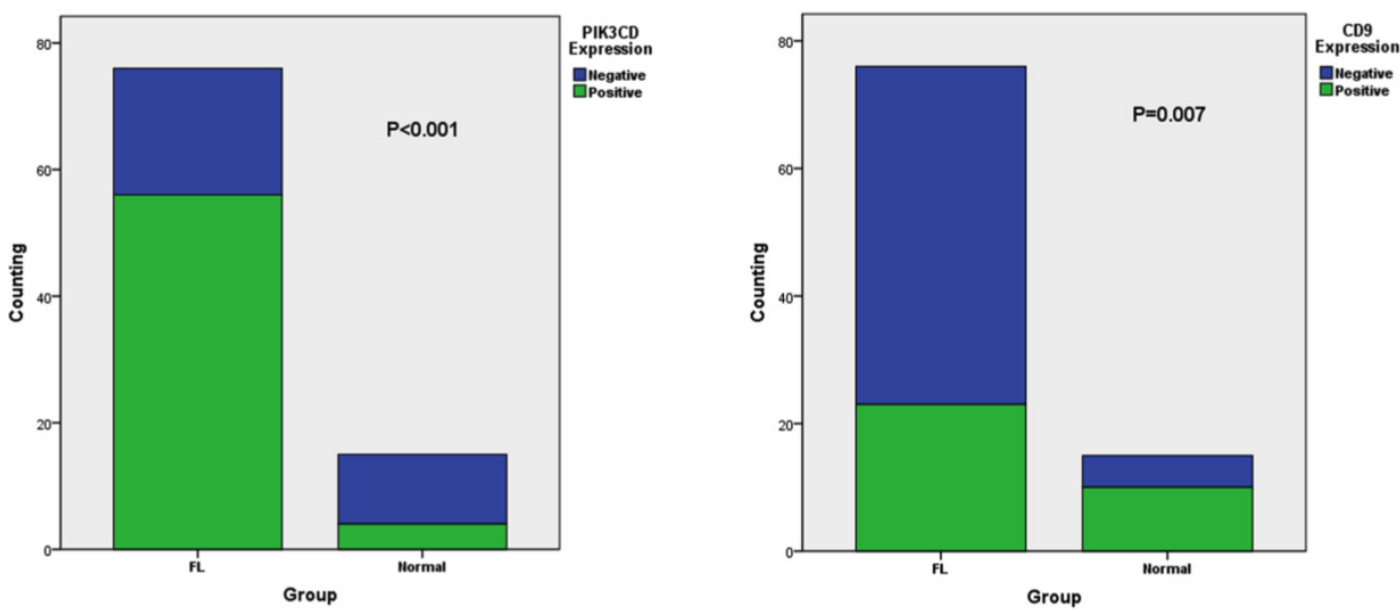

Fig. 3. The different expression of $C D 9$ (A) and PIK3CD (B) in FL and normal tissue
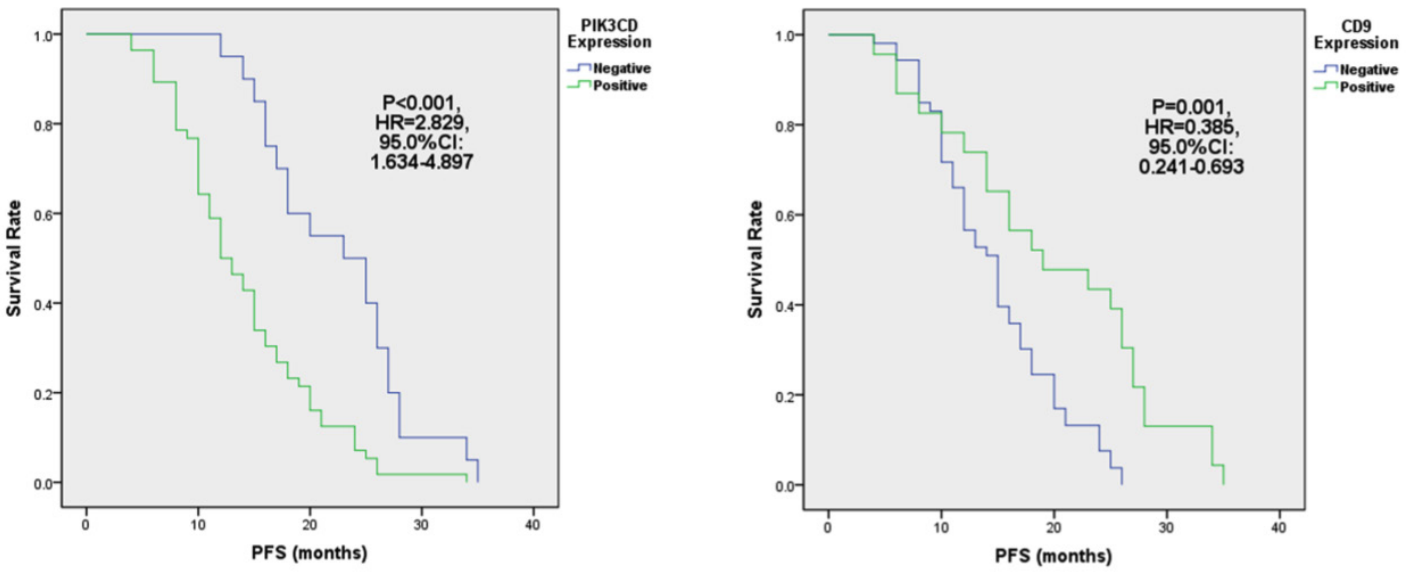

Fig.4. The expression of PIK3CD (A) and CD9 (B) is associated with PFS in FL.

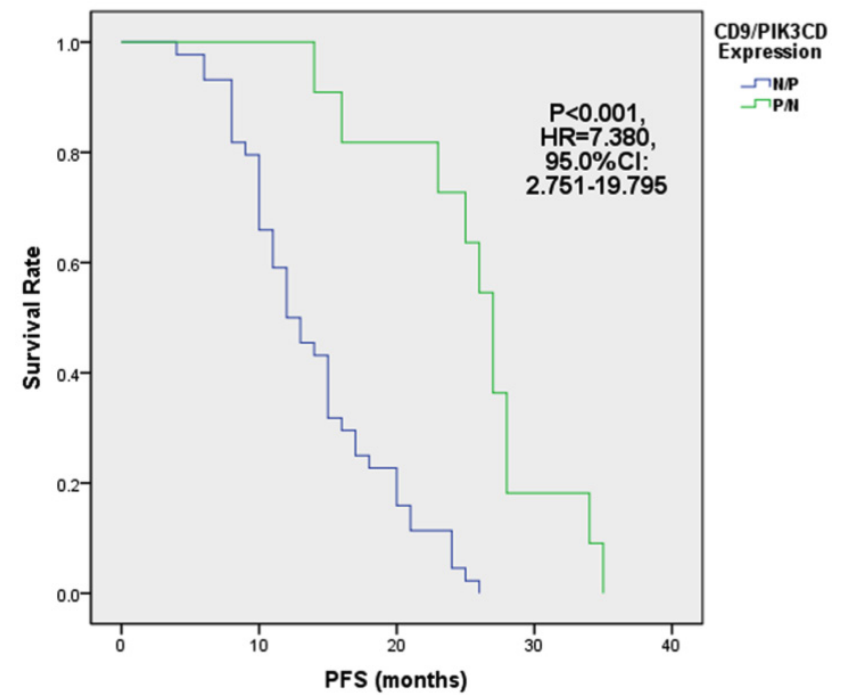

Fig. 5. FL patients with low CD9 and high PIK3CD had worse PFS.

\section{Discussion}

FL is the most common indolent B-cell lymphoma with various outcomes [2]. The heterogeneity of FL makes it difficult to predict its outcomes [43]. Therefore, prognostic markers are needed for better diagnosis and treatment. BCR signaling pathway play very important roles in B cell development, transformation and FL progression. Upon activation, BCR signaling transmits extracellular stimuli into cells via activation of PI3Ks, and subsequently phosphorylates Akt and Btk [44]. PI3K activation has also been observed in a FL animal model, but it has not been well studied in patient samples. Akt expression has been observed in all FL samples and phosphoryation of Akt occurred in 37\% (14/38) cases in a small cohort [24]. However, whether PI3K activation can be a prognostic marker is still not inclusive. PIK3CD is the predominant isoform of PI3Ks in B cells [17]. Here we examined PIK3CD expression in FL samples and found it was overexpressed in FL patients comparing with normal controls (Fig. 3A). Its overexpression was associated with high degrees of Ann Arbor stages (Table 1) and short PFS (Fig. 4A). These results proved that PI3K pathway play very important role in FL progression and can be used as a prognostic marker for FL. 
CD9 is a cell surface protein whose expression has been negatively correlated with prognostic outcomes in multiple cancers. It facilitates germinal center B cells interaction with FDCs, thus promote FDC-dependent survival of B cells. When B lymphoma cells progress and become FDC-independent, CD9 expression is reduced or lost [27]. Decreased expression of CD9 is more resistant to apoptosis. Nevertheless, whether CD9 expression contributes to FL progression is not well studied. In this study, we have shown that CD9 expression is reduced in FL patients (Fig. 3B) and patients with the reduced CD9 levels had higher degrees of Ann Arbor stages (Fig. 4B) and shorter PFS than those with high level CD9 (Table 1). Our results agree with previous discoveries and suggested that CD9 may play an important role in FL progression.

Surprisingly, we found CD9 overexpression is associated with bone marrow infiltration although in other solid cancers CD9 down-regulation was more often seen in metastatic lesions [28, 45, 46] (Table 3). The mechanisms behind this phenomenon remain elusive. It has been shown that CD9 positive B-acute lymphoblastic leukemia cells displayed cancer stem cell properties, including asymmetric division, self-renewal and drug resistance [29]. The association of CD9 and bone marrow infiltration may reflect this aspect of CD9's functions. It is possible that CD9 expression can predict different clinicopathological features due to its multiple facets of functions. On the other hand, the sample size of bone marrow infiltration FL is limited, so this phenomenon needs a further investigation.

We also found that PIK3CD expression is negatively correlated with CD9 levels in FL patients (Table 2 ), and PIK3CD(+)/CD9(-) patients have significantly shorter PFS (Fig. 5). These results suggested that PIK3CD and CD9 combination may be a better prognostic marker for FL. The regulatory networks of PIK3CD and CD9 are not well studied. In mast cells, CD9 is an alternative IL-16 receptor and can activate PI3K pathways [40]. Another study using mouse models have shown CD9 expression was markedly reduced in normal $B$ cells after Btk ablation, an important mediator of PI3K pathways [25]. Our observation does not agree with these results. The negative-correlation of CD9 and PIK3CD expression suggests that the regulatory network of these 2 proteins are complicated and their molecular functions in FL need further investigations.

In summary, we have shown that FL patients expressed high levels of PIK3CD and low levels of CD9. The upregulation of PIK3CD and downregulation of CD9 are associated with higher Ann Arbor stages and short PFS, respectively. However, CD9 expression may also promote FL bone marrow infiltration in Ann Arbor Stages IV patients. Concomitantly, PIK3CD expression negatively correlated with CD9 expression, and the CD9(-)/p110(+) group have the shortest PFS. These results suggested that the loss of CD9 and gain of PIK3CD increases the risk of FL patients recurrence, thus CD9 and PIK3CD are good predictors of tumor progression or aggressiveness of FL.

\section{Acknowledgements}

This study was supported by grant 81472636 from National Natural Science Foundation of China, the Department of Medical Oncology of the Third Affiliated Hospital of Harbin Medical University, Harbin, China, and the Tumor Research Institute of Heilongjiang, Harbin, China.

\section{Competing Interests}

The authors have declared that no competing interest exists.

\section{References}

1. Kuppers R. Mechanisms of B-cell lymphoma pathogenesis. Nature reviews Cancer. 2005; 5: 251-62. doi:10.1038/nrc1589.

2. Kridel R, Sehn LH, Gascoyne RD. Pathogenesis of follicular lymphoma. The Journal of clinical investigation. 2012; 122: 3424-31. doi:10.1172/JCI63186.

3. Salles G, Seymour JF, Offner F, et al. Rituximab maintenance for 2 years in patients with high tumour burden follicular lymphoma responding to rituximab plus chemotherapy (PRIMA): a phase 3, randomised controlled trial. Lancet. 2011; 377: 42-51. doi:10.1016/S0140-6736(10)62175-7.

4. Vidal L, Gafter-Gvili A, Salles G, et al. Rituximab maintenance for the treatment of patients with follicular lymphoma: an updated systematic review and meta-analysis of randomized trials. Journal of the National Cancer Institute. 2011; 103: 1799-806. doi:10.1093/jnci/djr418.

5. Al Khabori M, de Almeida JR, Guyatt GH, et al. Autologous stem cell transplantation in follicular lymphoma: a systematic review and meta-analysis. Journal of the National Cancer Institute. 2012; 104: 18-28. doi:10.1093/jnci/djr450.

6. Fend F, Quintanilla-Martinez L. Assessing the prognostic impact of immune cell infiltrates in follicular lymphoma. Haematologica. 2014; 99: 599-602. doi:10.3324/haematol.2014.104968.

7. Al-Tourah AJ, Gill KK, Chhanabhai M, et al. Population-based analysis of incidence and outcome of transformed non-Hodgkin's lymphoma. Journal of clinical oncology : official journal of the American Society of Clinical Oncology. 2008; 26: 5165-9. doi:10.1200/JCO.2008.16.0283.

8. Montoto S, Davies AJ, Matthews J, et al. Risk and clinical implications of transformation of follicular lymphoma to diffuse large B-cell lymphoma. Journal of clinical oncology : official journal of the American Society of Clinical Oncology. 2007; 25: 2426-33. doi:10.1200/JCO.2006.09.3260.

9. Lossos IS, Alizadeh AA, Diehn M, et al. Transformation of follicular lymphoma to diffuse large-cell lymphoma: alternative patterns with increased or decreased expression of c-myc and its regulated genes. Proceedings of the National Academy of Sciences of the United States of America. 2002; 99: 8886-91. doi:10.1073/pnas.132253599.

10. Craxton A, Otipoby KL, Jiang A, et al. Signal transduction pathways that regulate the fate of B lymphocytes. Advances in immunology. 1999; 73: 79-152.

11. Okkenhaug K, Vanhaesebroeck B. PI3K in lymphocyte development, differentiation and activation. Nature reviews Immunology. 2003; 3: 317-30. doi:10.1038/nri1056.

12. Koyasu S. The role of PI3K in immune cells. Nature immunology. 2003; 4: 313-9. doi:10.1038/ni0403-313.

13. Deane JA, Fruman DA. Phosphoinositide 3-kinase: diverse roles in immune cell activation. Annual review of immunology. 2004; 22: 563-98. doi:10.1146/annurev.immunol.22.012703.104721.

14. Curran E, Smith SM. Phosphoinositide 3-kinase inhibitors in lymphoma. Current opinion in oncology. 2014; 26: 469-75. doi:10.1097/CCO.0000000000000113.

15. Tuveson DA, Carter RH, Soltoff SP, et al. CD19 of B cells as a surrogate kinase insert region to bind phosphatidylinositol 3-kinase. Science. 1993; 260: 986-9. 
16. Xu Y, Fairfax K, Light A, et al. CD19 differentially regulates BCR signalling through the recruitment of PI3K. Autoimmunity. 2014; 47: 430-7. doi:10.3109/08916934.2014.921810.

17. Puri KD, Gold MR. Selective inhibitors of phosphoinositide 3-kinase delta: modulators of B-cell function with potential for treating autoimmune inflammatory diseases and B-cell malignancies. Frontiers in immunology. 2012; 3: 256. doi:10.3389/fimmu.2012.00256.

18. Chantry D, Vojtek A, Kashishian A, et al. p110delta, a novel phosphatidylinositol 3-kinase catalytic subunit that associates with p 85 and is expressed predominantly in leukocytes. The Journal of biological chemistry. 1997; 272: 19236-41.

19. Bilancio A, Okkenhaug K, Camps M, et al. Key role of the p110delta isoform of PI3K in B-cell antigen and IL-4 receptor signaling: comparative analysis of genetic and pharmacologic interference with p110delta function in B cells. Blood. 2006; 107: 642-50. doi:10.1182/blood-2005-07-3041.

20. Engelman JA, Luo J, Cantley LC. The evolution of phosphatidylinositol 3-kinases as regulators of growth and metabolism. Nature reviews Genetics. 2006; 7: 606-19. doi:10.1038/nrg1879.

21. Lannutti BJ, Meadows SA, Herman SE, et al. CAL-101, a p110delta selective phosphatidylinositol-3-kinase inhibitor for the treatment of B-cell malignancies, inhibits PI3K signaling and cellular viability. Blood. 2011; 117: 591-4. doi:10.1182/blood-2010-03-275305.

22. Herman SE, Gordon AL, Wagner AJ, et al. Phosphatidylinositol 3-kinase-delta inhibitor CAL-101 shows promising preclinical activity in chronic lymphocytic leukemia by antagonizing intrinsic and extrinsic cellular survival signals. Blood. 2010; 116: 2078-88. doi:10.1182/blood-2010-02-271171.

23. Seda V, Mraz M. B-cell receptor signalling and its crosstalk with other pathways in normal and malignant cells. European journal of haematology. 2014. doi:10.1111/ejh.12427.

24. Yahiaoui OI, Nunes JA, Castanier C, et al. Constitutive AKT activation in follicular lymphoma. BMC cancer. 2014; 14:565. doi:10.1186/1471-2407-14-565.

25. Lindvall JM, Blomberg KE, Berglof A, et al. Gene expression profile of B cells from Xid mice and Btk knockout mice. European journal of immunology. 2004; 34: 1981-91. doi:10.1002/eji.200324051.

26. Maecker HT, Todd SC, Levy S. The tetraspanin superfamily: molecular facilitators. FASEB journal : official publication of the Federation of American Societies for Experimental Biology. 1997; 11: 428-42.

27. Yoon SO, Zhang X, Freedman AS, et al. Down-regulation of CD9 expression and its correlation to tumor progression in B lymphomas. The American journal of pathology. 2010; 177: 377-86. doi:10.2353/ajpath.2010.100048.

28. Yoon SO, Lee IY, Zhang X, et al. CD9 may contribute to the survival of human germinal center $\mathrm{B}$ cells by facilitating the interaction with follicular dendritic cells. FEBS open bio. 2014; 4: 370-6. doi:10.1016/j.fob.2014.04.001.

29. Arita A, McFarland DC, Myklebust JH, et al. Signaling pathways in lymphoma: pathogenesis and therapeutic targets. Future oncology. 2013; 9: 1549-71. doi:10.2217/fon.13.113.

30. Hemler ME. Tetraspanin functions and associated microdomains. Nature reviews Molecular cell biology. 2005; 6: 801-11. doi:10.1038/nrm1736.

31. Hemler ME. Tetraspanin proteins mediate cellular penetration, invasion, and fusion events and define a novel type of membrane microdomain. Annual review of cell and developmental biology. 2003; 19: 397-422. doi:10.1146/annurev.cellbio.19.111301.153609.

32. Sugiura T, Berditchevski F. Function of alpha3beta1-tetraspanin protein complexes in tumor cell invasion. Evidence for the role of the complexes in production of matrix metalloproteinase 2 (MMP-2). The Journal of cell biology. 1999; 146: 1375-89.

33. Sawada S, Yoshimoto M, Odintsova E, et al. The tetraspanin CD151 functions as a negative regulator in the adhesion-dependent activation of Ras. The Journal of biological chemistry. 2003; 278: 26323-6. doi:10.1074/jbc.C300210200.

34. Miyake M, Nakano K, Ieki Y, et al. Motility related protein 1 (MRP-1/CD9) expression: inverse correlation with metastases in breast cancer. Cancer research. 1995; 55: 4127-31.

35. Wang JC, Begin LR, Berube NG, et al. Down-regulation of CD9 expression during prostate carcinoma progression is associated with CD9 mRNA modifications. Clinical cancer research : an official journal of the American Association for Cancer Research. 2007; 13: 2354-61. doi:10.1158/1078-0432.CCR-06-1692.

36. Houle CD, Ding XY, Foley JF, et al. Loss of expression and altered localization of KAI1 and CD9 protein are associated with epithelial ovarian cancer progression. Gynecologic oncology. 2002; 86: 69-78.

37. Won WJ, Kearney JF. CD9 is a unique marker for marginal zone B cells, B1 cells, and plasma cells in mice. Journal of immunology. 2002; 168: 5605-11.

38. Yang $\mathrm{H}$, Shen $\mathrm{C}$, Zhang B, et al. Expression and clinicopathological significance of CD9 in gastrointestinal stromal tumor. Journal of Korean medical science. 2013; 28: 1443-8. doi:10.3346/jkms.2013.28.10.1443.

39. Relander T, Johnson NA, Farinha P, et al. Prognostic factors in follicular lymphoma. Journal of clinical oncology : official journal of the American Society of Clinical Oncology. 2010; 28: 2902-13. doi:10.1200/JCO.2009.26.1693.

40. Qi JC, Wang J, Mandadi S, et al. Human and mouse mast cells use the tetraspanin CD9 as an alternate interleukin-16 receptor. Blood. 2006; 107: 135-42. doi:10.1182/blood-2005-03-1312.

41. Jager $U$, Fridrik $M$, Zeitlinger $M$, et al. Rituximab serum concentrations during immuno-chemotherapy of follicular lymphoma correlate with patient gender, bone marrow infiltration and clinical response. Haematologica. 2012; 97: 1431-8. doi:10.3324/haematol.2011.059246.

42. Che YQ, Liu P, Wang $Y$, et al. Bcl-2/IgH expression in minimal bone marrow infiltration by follicular lymphoma cells. Molecular medicine reports. 2012; 5: 383-7. doi:10.3892/mmr.2011.663.

43. Takata K, Miyata-Takata T, Sato Y, et al. Pathology of follicular lymphoma. Journal of clinical and experimental hematopathology : JCEH. 2014; 54: 3-9.

44. Garcia-Martinez JM, Wullschleger S, Preston G, et al. Effect of PI3K- and mTOR-specific inhibitors on spontaneous B-cell follicular lymphomas in PTEN/LKB1-deficient mice. British journal of cancer. 2011; 104: 1116-25. doi:10.1038/bjc. 2011.83.

45. van Spriel AB. Tetraspanins in the humoral immune response. Biochemical Society transactions. 2011; 39: 512-7. doi:10.1042/BST0390512.

46. Zoller M. Tetraspanins: push and pull in suppressing and promoting metastasis. Nature reviews Cancer. 2009; 9: 40-55. doi:10.1038/nrc2543. 